\title{
Severity and distribution of cartilage damage and bone marrow edema in the patellofemoral and tibiofemoral joints in knee osteoarthritis determined by MRI
}

\author{
BAOMING DONG ${ }^{1}$, YANLIANG KONG ${ }^{2}$, LEI ZHANG ${ }^{3}$ and YONGQIAN QIANG ${ }^{1}$ \\ ${ }^{1}$ Department of Radiology, The First Affiliated Hospital of Xi'an Jiaotong University Medical College, Xi'an, \\ Shaanxi 710061; ${ }^{2}$ Department of Radiology, Tongchuan People's Hospital, Tongchuan, Shaanxi 727000; ${ }^{3}$ Department of \\ Radiology, The Second Affiliated Hospital of Xi'an Jiaotong University Medical College, Xi'an, Shaanxi 710054, P.R. China
}

Received December 17, 2015; Accepted December 23, 2016

DOI: $10.3892 /$ etm.2017.4190

\begin{abstract}
The aim of the present study was to analyze the distribution and severity of cartilage damage $(\mathrm{CD})$ and bone marrow edema (BME) of the patellofemoral and tibiofemoral joints (PFJ and TFJ, respectively) in patients with knee osteoarthritis (OA), and to determine whether a correlation exists between $\mathrm{BME}$ and $\mathrm{CD}$ in knee OA, using magnetic resonance imaging (MRI). Forty-five patients diagnosed with knee OA (KOA group) and 20 healthy individuals (control group) underwent sagittal multi-echo recalled gradient echo sequence scans, in addition to four conventional MR sequence scans. Knee joints were divided into 15 subregions by the whole-organ MRI scoring method. MRIs of each subregion were analyzed for the presence of $\mathrm{CD}, \mathrm{CD}$ score and BME score. The knee joint activity functional score was determined using the Western Ontario and McMaster Universities Arthritis Index (WOMAC) in the KOA group. Statistical analyses were used to compare the $\mathrm{CD}$ incidence; $\mathrm{CD}$ score and BME score between the PFJ and TFJ. Whether a correlation existed among body mass index, BME score, WOMAC pain score and CD score was also examined. Among the 675 subregions analyzed in the KOA group, 131 exhibited CD (CD score, 1-6). These 131 subregions were primarily in the PFJ $(80 / 131,61.07 \%)$, with the remainder in the TFJ $(51 / 131,38.93 \%)$. Thirty-three subregions had a CD score of 1, including 24 PFJ subregions (72.73\%) and 9 TFJ subregions (27.27\%). Among the 103 subregions with BME, the PFJ accounted for 60 (58.25\%) and the TFJ for 43 (41.75\%). A significant positive correlation was found between the BME and $\mathrm{CD}$ scores. In conclusion, $\mathrm{CD}$ and $\mathrm{BME}$ occurred earlier
\end{abstract}

Correspondence to: Dr Yongqian Qiang, Department of Radiology, The First Affiliated Hospital of Xi'an Jiaotong University Medical College, 277 Yanta West Road, Xi'an, Shaanxi 710061, P.R. China E-mail: imagingqyq@163.com

Key words: patellofemoral joint, cartilage damage, multi-echo recalled gradient echo, semi-quantitative and more often in the PFJ compared to the TFJ in knee OA, and $\mathrm{BME}$ is an indirect sign of CD.

\section{Introduction}

With the increasing age and obesity rate of the world's population (1), the incidence of knee osteoarthritis (OA) is increasing. Knee OA is the main cause of disability and functional limitation among elderly individuals $(2,3)$. Radiological and physical examinations represent noninvasive methods for OA diagnosis. However, ordinary X-ray can only identify a change of bone structure under the articular surface and joint space narrowing (4). By contrast, high-resolution magnetic resonance imaging (MRI) can be used to detect changes in bone structure, cartilage damage (CD), meniscal tears, synovitis, subchondral cysts, bone attrition, ligament trauma and bone marrow edema (BME) $(5,6)$.

The knee joint is composed of the patellofemoral joint (PFJ) and the tibiofemoral joint (TFJ). Previous studies have mainly focused on TFJ-OA, whereas PFJ-OA has been less studied (7). A previous study suggested that PFJ-OA is at least as common as TFJ-OA (8). Alone or in combination with TFJ-OA, PFJ-OA may account for up to $65 \%$ of cases of symptomatic knee OA. Therefore, more attention must be paid to the changes that occur in PFJ-OA (9). In particular, PFJ-OA is closely associated with knee joint pain and dysfunction. Which region of the knee is the first to be affected by OA and the most likely to lead to knee pain are clinically important questions for clinicians (10).

The aims of the present study were as follows: i) To compare the distribution and severity of CD and BME between the PFJ and TFJ in knee OA by MRI; and ii) to determine whether a correlation exists between BME and CD in knee OA.

\section{Materials and methods}

Participants and study design. Approval of the present study was obtained from the Institutional Review Board of the First Affiliated Hospital of Xi'an Jiaotong University Medical College (Xi'an, China). All participants signed informed consent forms prior to participating. Between December 2013 

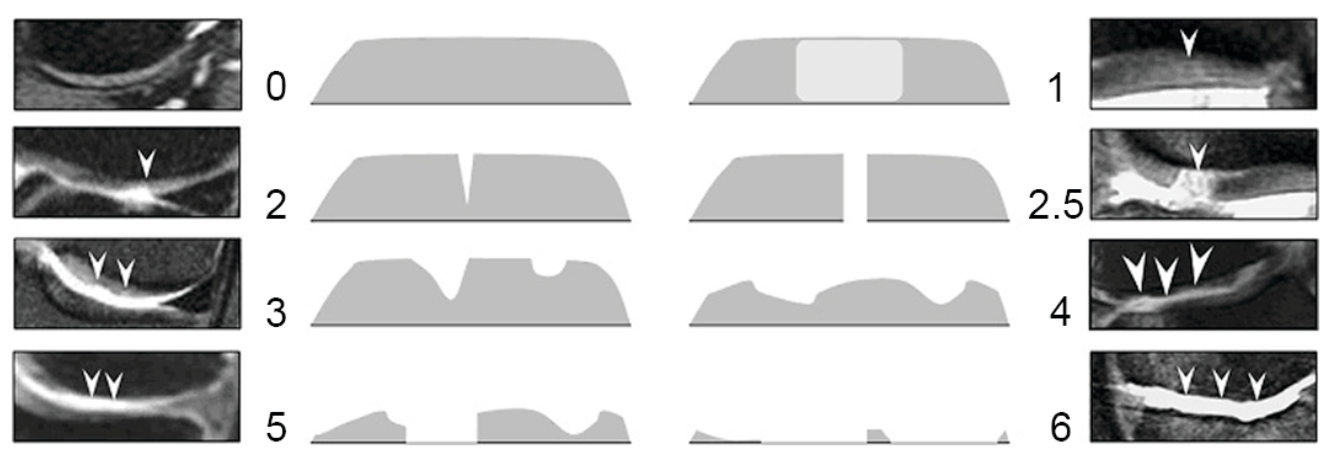

Figure 1. Cartilage signal intensity and morphology were scored using an 8-point scale from Hayashi et al (13): 0, normal thickness and signal; 1 , normal thickness but increased signal on fat-suppression proton density-weighted turbo spin echo image; 2.0 , partial-thickness focal defect $<1 \mathrm{~cm}$ in greatest width; 2.5, full-thickness focal defect $<1 \mathrm{~cm}$ in greatest width; 3, multiple areas of partial-thickness (grade 2.0) defects intermixed with areas of normal thickness, or a grade 2.0 defect $>1 \mathrm{~cm}$ comprising $<75 \%$ of the subregion; 4 , diffuse ( $\geq 75 \%$ of the subregion) partial-thiwness loss; 5 , multiple areas of full-thickness loss (grade 2.5), or a grade 2.5 lesion $>1 \mathrm{~cm}$ comprising $<75 \%$ of the subregion; 6 , diffuse ( $\geq 75 \%$ of the subregion) full-thickness loss. Arrowheads indicate cartilage damage degree from 0 to 6 standard atlas in cases.

and December 2014, 45 individuals with knee OA who underwent MR examination at The First Affiliated Hospital of Xi'an Jiaotong University Medical College (Xi'an, China) were selected for the present study (KOA group). During the same period, 20 healthy volunteers were selected from inside and outside the hospital.

The 1995 revised diagnostic criteria (11) for knee OA of the American College of Rheumatism Association are as follows: i) Knee pain of at least 1 month's duration, ii) bone friction with knee activity, iii) morning stiffness lasting $\leq 30 \mathrm{~min}$, iv) age $>38$ years, and v) presence of bone osteophytes. Participants who met criteria i-iv, criteria i, ii and $v$ or criteria $\mathrm{i}$, iv and $\mathrm{v}$ were diagnosed with knee OA and included in the study. Exclusion criteria for the KOA group were as follows: Diagnosis with ankylosing spondylitis, rheumatoid arthritis, reactive arthritis, psoriatic arthritis or any other type of chronic immune disease, a history of knee trauma or surgery, treatment with systemic corticosteroids, intra-articular injections or drugs for other bone-associated diseases, contraindication for MR examination and refusal to participate.

For all participants, body height and weight were recorded and the body mass index (BMI) was calculated as the weight divided by height squared $\left(\mathrm{kg} / \mathrm{m}^{2}\right)$. The Western Ontario and McMaster Universities Osteoarthritis Index (WOMAC) was used to evaluate knee joint function for subjects in the KOA group.

MR scan sequence and parameters. All 65 participants underwent MR scans with the GE OPTIMA fiber MR 360 1.5 T MRI machine (GE Healthcare Systems, Pittsburgh, PA, USA) using a phased array knee coil. MR sequences included the following four conventional knee scan sequences: Oblique Sagittal (OSAG) T2 proton density-weighted (PD) fat-suppression (FS) fast spin echo (FSE) imaging, OSAG-T1-FSE, coronary T2 PD-FS-FSE and cross-sectional T2 PD-FS-FSE imaging. In addition, the OSAG multi-echo gradient imaging sequence was used, with the following scan parameters: Repetition time, 529; echo time, 16; echo train length, 6; echo sequence, 1; slice thickness (ST), $4 \mathrm{~mm}$; slice spacing (SS), $0.4 \mathrm{~mm}$; spin echo, 16; filed of vies, 18x18;
MATRIX, 256x256; NEX, 2; bandwidth, 63; and acquire time (TA), 4:35.

Semi-quantitative assessment of MR images. By applying the whole-organ MRI scoring (WORMS) method (12), knee joint cartilage was divided in 15 anatomic subregions on MRI (13). Knee joint cartilage in the sagittal position was divided into subregions designated as femoral or tibial, lateral or medial, and central, posterior or anterior. The patella was divided into medial and lateral subregions in the axial plane. Medial and lateral regions of the femoral trochlea cartilage were defined. The subspinous subregion of the tibia has no cartilage and was excluded from analysis. The PFJ comprises five subregions: The patella medial and lateral subregions, and the femoral medial, lateral anterior and lateral trochlea subregions. The TFJ comprises 10 subregions: The femoral medial, lateral central and posterior subregions, and the tibial medial, lateral, anterior, central and posterior subregions.

Evaluation of $C D$ and BME by semi-quantitative MRI. MRIs of the knee joint cartilage were inputted into the Centricity DICOM Viewer 3.1.3 post-processing workstation (GE Healthcare Life Sciences, Chalfont, UK). Two trained comprehensive radiologists who were blinded to the patient groups assessed the cartilage morphology (i.e., damaged or undamaged), CD score (described in detail in Fig. 1) and BME score for each of the 15 WORMS subregions. For subregions with $C D$, the highest $\mathrm{CD}$ score for the subregion was recorded. BME with high signal intensity was scored as follows (10): 0 , Normal bone marrow without edema; 1 , mild edema of $<25 \%$ of the area (Fig. 2A); 2, moderate edema of $25-50 \%$ of the area (Fig. 2B); and 3, severe edema of $\geq 50 \%$ of the area (Fig. 2C). In the case of disagreement between observers, a consensus was achieved through discussion.

Statistical analysis. Excel 2013 (Microsoft Corp., Redmont, WA, USA) and the SPSS 18.0 statistical software package (SPSS, Inc., Chicago, IL, USA) were used for data entry and statistical analysis. Values are expressed as the mean value \pm standard deviation. Chi-squared tests were used to 
Table I. Demographic characteristics of the study population.

\begin{tabular}{lcc}
\hline Characteristic & $\begin{array}{c}\text { Control group } \\
(\mathrm{n}=20)\end{array}$ & $\begin{array}{c}\text { KOA group } \\
(\mathrm{n}=45)\end{array}$ \\
\hline Gender (male/female) & $14 / 6$ & $12 / 33$ \\
Age (years) & $35.0 \pm 10.8$ & $50.0 \pm 13.3$ \\
Body mass index $\left(\mathrm{kg} / \mathrm{m}^{2}\right)$ & $23.5 \pm 3.0$ & $24.4 \pm 3.2$ \\
Left/right knee & $12 / 8$ & $19 / 26$ \\
\hline
\end{tabular}

Values are expressed as the mean \pm standard deviation or the number as applicable. KOA, knee osteoarthritis.

compare the articular CD and BME scores between the PFJ and TFJ. Cartilage morphology, CD score and BME score were compared between the KOA and control groups by the Student's t-test. Multivariate linear regression and Pearson's correlation coefficient were used to assess whether a correlation existed in the KOA group among the $\mathrm{CD}$ score, BME score, WOMAC pain score and BMI. $\mathrm{P}<0.05$ was considered to indicate a statistically significant difference. A positive correlation was defined as an $\mathrm{R}>0$.

\section{Results}

Demographic data. Table I summarizes the demographic information for the KOA and control groups. There were no significant differences between the two groups in terms of gender distribution or BMI (BMI, $\mathrm{t}=1.048, \mathrm{P}=0.298)$, while the groups differed in terms of age $(\mathrm{t}=4.498, \mathrm{P}<0.001)$.

Distribution and characteristics of $C D$ in the KOA group. Among the 675 subregions examined in the KOA group, there were 131 subregions with CD scores of 1-6. A CD score of 2.5 frequently indicated more serious damage. However, as no participant had a score of exactly 2.5 , subregions were divided into those having CD scores of 1-3 (48 subregions) or 4-6 (83 subregions). Among the 131 subregions with $\mathrm{CD}, 80$ subregions (61.07\%) were in the PFJ and 51 subregions (38.93\%) were in the TFJ $\left(\chi^{2}=56.267, \mathrm{P}<0.001\right)$. Two instances of CD were in the femoral lateral trochlea subregion. Thirty-three subregions had a CD score of 1 , including 24 subregions $(72.73 \%)$ in the PFJ and 9 subregions $(27.27 \%)$ in the TFJ $\left(\chi^{2}=24.230, P<0.001\right.$; Fig. 3$)$.

$B M E$ distribution and characteristics in the KOA group. BME was identified in 103 of the 675 examined subregions, with a BME score of 1 in 54 subregions, 2 in 34 subregions and 3 in 54 subregions. Among the 103 subregions with a BME score of $1-3,60$ subregions $(58.25 \%)$ were in the PFJ and 43 subregions $(41.75 \%)$ were in the TFJ $\left(\chi^{2}=33.961\right.$, $\mathrm{P}<0.001)$. There were 54 'early' BME lesions (BME score, 1 ), which were more frequently found in the PFJ (35 subregions, 64.8\%) compared to the TFJ (19 subregions, 35.2\%) $\left(\chi^{2}=26.178, \mathrm{P}<0.001\right)$.

Correlation among BMI and the CD, BME and WOMAC pain scores. Table II reports the correlation coefficients among
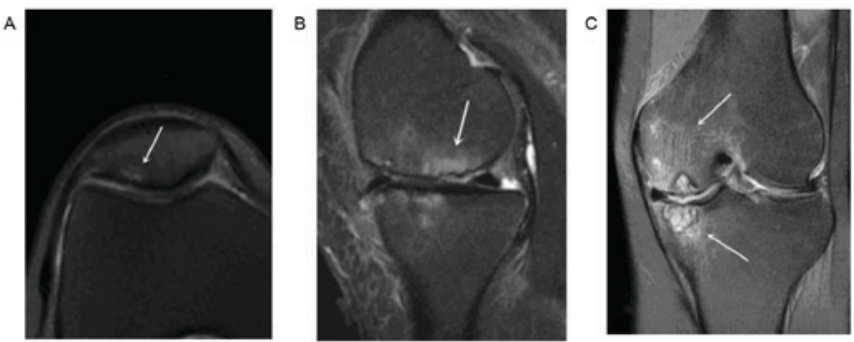

Figure 2. (A) Cross-sectional T2 PD-FS-FSE image with BME score of 1. (B) Sagittal T2 PD-FS-FSE image with BME score of 2. (C) Coronary T2 PD-FS-FSE image with a BME score of 3. Arrows indicate BME in each image. On the sagittal PD-FS-FSE image, the subchondral area on the boundary did not have a clear signal. BME, bone marrow edema; PD-FS-FSE, proton density-weighted fat-suppression fast spin echo.
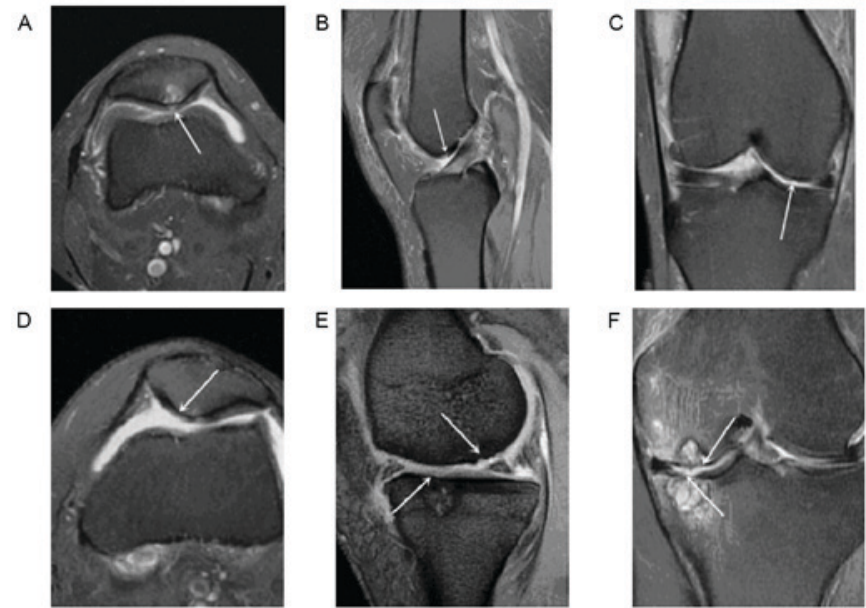

Figure 3. (A) Cross-sectional T2 PD-FS-FSE image of the right knee of a 65-year-old woman. The arrow indicates patellar CD with a score of 1 . (B) Sagittal T2 PD-FS-FSE image of the left knee of a 56-year-old man. The arrow indicates lateral trochlear CD with a score of 2. (C) Coronary T2 PD-FS-FSE image of the right knee of a 60-year-old woman. The arrow indicates lateral condyle CD with a score of 3. (D) Cross-sectional T2 PD-FS-FSE image of the right knee of a 66-year-old woman. The arrow indicates patellar CD with a score of 4. (E) Sagittal multi-echo gradient T2-weighted image of the left knee of a 62-year-old man. The arrows indicate TFJ CD with a score of 5. (F) Coronary T2 PD-FS-FSE image of the left knee of a 61-year-old woman. The arrow indicates TFJ CD with a score of 6. CD, cartilage damage; PD-FS-FSE, proton density-weighted fat-suppression fast spin echo; TFJ, tibiofemoral joint.

BMI, WOMAC pain score, BME score and CD score. There was a significant positive correlation between the BME and CD scores, which was confirmed by a strong linear correlation in the scatter diagram $(\mathrm{P}<0.001, \mathrm{R}=0.736$; Fig. 4).

\section{Discussion}

In the present study, the incidence of PFJ-OA was significantly higher than that of TFJ-OA. The incidence of early-stage CD without obvious morphological alterations (CD score, 1) was significantly higher in the PFJ compared to the TFJ. In the control group, regions of $\mathrm{CD}$ with a high signal intensity were not found on MRI. These findings are similar to those of Hayashi et al (13), who used MRI to examine the distribution and incidence of cartilage lesions (damage grade $>2$ ) in different knee joints of 696 elderly OA patients without 
Table II. Correlation among BMI and BME, WOMAC pain and CD scores.

\begin{tabular}{|c|c|c|c|c|}
\hline Parameter & BME score & WOMAC pain score & BMI & CD score \\
\hline \multicolumn{5}{|l|}{ BME score } \\
\hline Pearson correlation & 1 & -0.036 & 0.132 & 0.736 \\
\hline P-value & - & 0.825 & 0.389 & $<0.001^{\mathrm{a}}$ \\
\hline $\mathrm{N}$ & 45 & 41 & 45 & 45 \\
\hline \multicolumn{5}{|l|}{ WOMAC pain score } \\
\hline Pearson correlation & -0.036 & 1 & -0.129 & -0.028 \\
\hline P-value & 0.825 & - & 0.423 & 0.864 \\
\hline $\mathrm{N}$ & 41 & 41 & 41 & 41 \\
\hline \multicolumn{5}{|l|}{ BMI } \\
\hline Pearson correlation & 0.132 & -0.129 & 1 & 0.103 \\
\hline P-value & 0.389 & 0.423 & - & 0.501 \\
\hline $\mathrm{N}$ & 45 & 41 & 45 & 45 \\
\hline \multicolumn{5}{|l|}{ CD score } \\
\hline Pearson correlation & 0.736 & -0.028 & 0.103 & 1 \\
\hline P-value & $<0.001^{\mathrm{a}}$ & 0.864 & 0.501 & - \\
\hline $\mathrm{N}$ & 45 & 41 & 45 & 45 \\
\hline
\end{tabular}

${ }^{a}$ Two-tailed $\mathrm{P}<0.01$. P-values represent the mutual correlation of WOMAC, BME, CD and BMI and indicate that only BME and CD are associated. N, number of patients; WOMAC, Western Ontario and McMaster Universities Arthritis Index; BME, bone marrow edema; CD, cartilage damage; BMI, body mass index.

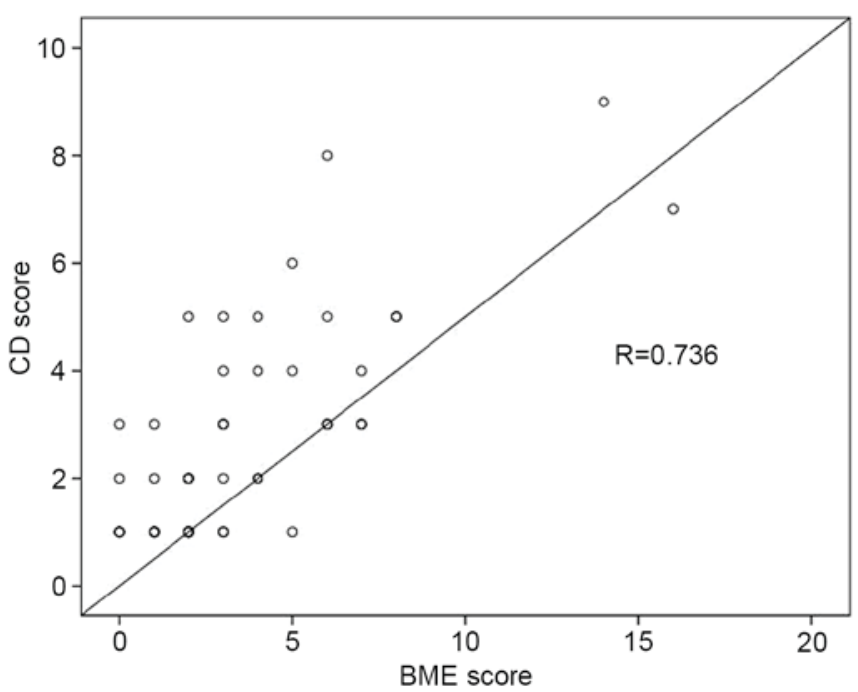

Figure 4. Scatter diagram of BME and CD scores. There was a significant positive correlation $(\mathrm{P}<0.001 ; \mathrm{R}=0.736)$. $\mathrm{BME}$, bone marrow edema; $\mathrm{CD}$, cartilage damage.

X-ray evidence of disease. The incidence of cartilage lesions was highest in the patellar subregions (medial, 47.7\%; lateral, $29.9 \%$ ) followed by the femoral subregions (medial anterior, 24.0\%; medial central, 26.5\%).

Duncan et al (14) stated that articular cartilage of the femoral anterior subregion, particularly in the PFJ, should not be ignored in the assessment of OA. The role of the PFJ in knee OA symptoms may be even more important than the role of the TFJ (15). Results from a longitudinal cohort study (16) and an animal model study (17) indicated that the PFJ is more susceptible to degeneration than the TFJ for cases with decreased muscle strength of the quadriceps femoris. Another study stated that isolated PFJ-OA may lead to obvious functional limitation of the knee joint (9). According to a recent community survey, osteophytes were found at higher rates in cases of PFJ-OA than TFJ-OA during plain film examination (218/334 vs. $184 / 334 ; \mathrm{P}<0.01)(18)$.

Hunter et al (19) considered the volume reduction of patellar cartilage to be more closely associated with knee joint pain than the reduction of tibial cartilage. In isolated PFJ-OA or TFJ-OA of 407 knee joints, 193 (47\%) knee joints had isolated damage of the PFJ articular cartilage, compared to $112(28 \%)$ with isolated damage of the TFJ cartilage (10). Another 25\% of knee joints had no CD. Peat et al (20) found $236(32 \%)$, $178(24 \%), 30(4 \%)$ and $301(40 \%)$ cases with no radiological features of OA, isolated PFJ-OA, isolated TFJ-OA, and combined PFJ-and TFJ-OA.

Considering the results of the present and previous studies, it is indicated that PFJ articular CD occurs earlier and more frequently than TFJCD in patients with knee OA. There are several possible reasons for these findings. First, the patellar cartilage is in direct contact with cartilage of the femoral anterior subregion in the PFJ. The PFJ does not have a meniscus that can buffer the force in the knee. Furthermore, the areas of activity and mobility are large in the PFJ. By contrast, the TFJ has a double protective structure of articular cartilage and meniscus. Thus, TFJ degeneration occurs later than PFJ degeneration.

Second, PFJ-OA has a unique pathogenesis, with the surface pressure distribution and size of the joint having important roles (2). When the PFJ is in the horizontal plane of translational motion (e.g., a person is standing with their 
knees unbent), the joint does not experience stress from body weight. However, the patellar cartilage surface experiences mechanical pressure-associated friction from $0^{\circ}$ and $90^{\circ}$ during activities involving bending or direct pulling of the quadriceps tendon and patellar ligament. Moreover, the PFJ has a physiological leverage function, in which the mechanical pressure of the knee is concentrated at a point on the PFJ surface. During knee weight-bearing activities, the PFJ surface bears the summation vector of force from the femoral quadriceps and patellar ligament. Any increase in this force will aggravate the PFJ reaction force, which can reach levels of three times the body weight during stair-climbing and seven to eight times the body weight during squatting activities $(21,22)$. Under repeated pressure action, the PFJ cartilage can experience stress overload, internal damage, fracture of the collagen fiber frame and local edema, with damage spreading outward from the cartilage.

High signals for the internal cartilage morphology were obtained on T2-weighted images, but the external morphology and surface changes of the cartilage were not obvious from the images. The damaged cartilage surface generally appears coarse, with a temporary increase in the cartilage thickness, resulting in softening, fibrosis and fissure of the cartilage surface. As the cartilage cells themselves have limited repair ability, degeneration of the cartilage morphology is inevitable under these conditions. Internal degeneration of the cartilage may occur due to knee overuse or locally uneven mechanical pressure. The femoral cartilage has a higher overall coefficient of compressive deformation and lower permeability than the patellar cartilage (23). The BMI is a critical factor in determining the cartilage pressure. Excess weight places stress on the joints and quickly wears down the cartilage. Numerous studies have reported that CD is more severe and occurs more frequently in obese adult female populations compared to ageand gender-matched (female) normal weight groups (24-26). Felson (27) found that age-matched elderly obese women had greater progression of knee OA compared to women of a normal weight, which was proposed to be due to abnormal mechanical stress of the knee joint.

Subchondral bone changes are crucial pathological changes in OA. Under conditions of CD, MRI shows local BME under the damaged cartilage. Articular cartilage has no pain receptors (28), whereas BME is rich in pain fibers. Numerous studies have proposed that BME may be the underlying cause of pain in OA and epidemiological studies have demonstrated a clear association between pain and osteophytes $(1,29,30)$. Moreover, an association between pain and sclerosis of subchondral bone has been indicated (4). Cartilage nutrition, particularly of deep-layer cartilage, is provided by the subchondral bone and synovial fluid. Microvascular changes may cause an imbalance in cartilage nutrition, with subsequent damage. Mild bone damage (microdamage) and BME are the earliest pathological changes of the subchondral bone, and these changes can lead to bone pain. Accumulation of extracellular fluid in BME leads to increased bone internal pressure and stimulation of the pain receptors. Further development of BME can cause hyperplasia of the subchondral bone and thinning/stripping of cartilage until the compact bone of the articular surface is exposed.

Numerous studies have considered BME to be associated with cartilage degeneration and the development of OA (31-34).
For instance, Baranyay et al (32) found that large BME lesions were more common in patients with knee pain than in those without. In the present study, statistical analysis showed that $\mathrm{CD}$ in $15 \mathrm{knee}$ joint subregions was positively correlated with $\mathrm{BME}$ around the cartilage lesions $(\mathrm{R}=0.736, \mathrm{P}<0.001)$. If morphological changes are regarded as direct signs of $\mathrm{CD}$, $\mathrm{BME}$ can be considered as an indirect sign of CD.

The WOMAC scoring system is used to assess pain, stiffness, feeling and physical function in patients with knee and hip OA (35). The higher the WOMAC score, the more severe the clinical manifestations of knee OA. The WOMAC is a clinically practical and important means of evaluation for knee pain, which has been extensively validated and tested. In the present study, the correlation coefficient between the WOMAC and CD scores was not statistically significant. One possible reason for the lack of correlation is that the WOMAC score does not reflect microscopic changes in CD, such as morphological changes of the cartilage. Severe CD involves the subchondral bone, which contains pain fibers, and affects the activity of the knee joint. In this case, the WOMAC score would be expected to correlate with the CD score.

In contrast to numerous studies demonstrating that cartilage volume decreases with increasing BMI $(36,37)$, the present study revealed no significant correlation between $\mathrm{BMI}$ and the CD score. One possible reason for this finding is that the Chinese average BMI differs from standard BMI designations. In the Chinese standard, individuals with a $\mathrm{BMI}<18.5 \mathrm{~kg} / \mathrm{m}^{2}$ are defined as underweight, those with a BMI of $18.5-23.9 \mathrm{~kg} / \mathrm{m}^{2}$ are considered to be of normal weight, those with $24-27.9 \mathrm{~kg} / \mathrm{m}^{2}$ are overweight and those with a BMI $>28 \mathrm{~kg} / \mathrm{m}^{2}$ are obese. Therefore, the correlation between $\mathrm{BMI}$ and $\mathrm{CD}$ in the present study may not be comparable to that determined by previous studies.

The present study had certain limitations. First, due to the small number of subjects, stratified data analysis could not be performed. The second limitation was the lack of an arthroscopic control. Although arthroscopic data are more intuitive than MR data, arthroscopy is an invasive procedure and is less commonly performed than MRI. Finally, no comparative continuous longitudinal tracking study has been made.

In conclusion, the PFJ has an important role in cartilage degeneration of the knee joint. Damage occurs earlier and more often in PFJ cartilage compared to TFJ cartilage during knee OA. Furthermore, BME occurs synchronously with cartilage damage in knee OA. Future research should include a longitudinal quantitative study of the earliest high-intensity signal to appear on MRI in the articular cartilage during OA-associated degeneration of the PFJ and TFJ.

\section{References}

1. Guermazi A, Niu J, Hayashi D, Roemer FW, Englund M, Neogi T, Aliabadi P, McLennan CE and Felson DT: Prevalence of abnormalities in knees detected by MRI in adults without knee osteoarthritis: Population based observational study (Framingham Osteoarthritis Study). BMJ 345: e5339, 2012.

2. Crema MD, Guermazi A, Sayre EC, Roemer FW, Wong H, Thorne A, Singer J, Esdaile JM, Marra MD, Kopec JA, et al: The association of magnetic resonance imaging (MRI)-detected structural pathology of the knee with crepitus in a population-based cohort with knee pain: The MoDEKO study. Osteoarthritis Cartilage 19: 1429-1432, 2011. 
3. Bedson J and Croft PR: The discordance between clinical and radiographic knee osteoarthritis: A systematic search and summary of the literature. BMC Musculoskelet Disord 9: 116, 2008.

4. Amin S, LaValley MP, Guermazi A, Grigoryan M, Hunter DJ, Clancy M, Niu J, Gale DR and Felson DT: The relationship between cartilage loss on magnetic resonance imaging and radiographic progression in men and women with knee osteoarthritis. Arthritis Rheum 52: 3152-3159, 2005.

5. Sowers M, Karvonen-Gutierrez CA, Jacobson JA, Jiang Y and Yosef M: Associations of anatomical measures from MRI with radiographically defined knee osteoarthritis score, pain, and physical functioning. J Bone Joint Surg Am 93: 241-251, 2011.

6. Guermazi A, Roemer FW and Hayashi D: Imaging of osteoarthritis: Update from a radiological perspective. Curr Opin Rheumatol 23: 484-491, 2011.

7. Farrokhi S, Piva SR, Gil AB, Oddis CV, Brooks MM and Fitzgerald GK: Association of severity of coexisting patellofemoral disease with increased impairments and functional limitations in patients with knee osteoarthritis. Arthritis Care Res (Hoboken) 65: 544-551, 2013

8. Stefanik JJ, Niu J, Gross KD, Roemer FW, Guermazi A and Felson DT: Using magnetic resonance imaging to determine the compartmental prevalence of knee joint structural damage. Osteoarthritis Cartilage 21: 695-699, 2013.

9. Duncan R, Peat G, Thomas E, Wood L, Hay E and Croft P: Does isolated patellofemoral osteoarthritis matter? Osteoarthritis Cartilage 17: 1151-1155, 2009.

10. Stefanik JJ, Neogi T, Niu J, Roemer FW, Segal NA, Lewis CE, Nevitt M, Guermazi A and Felson DT: The diagnostic performance of anterior knee pain and activity-related pain in identifying knees with structural damage in the patellofemoral joint: The multicenter osteoarthritis study. J Rheumatol 41: 1695-1702, 2014

11. Singh AK, Kalaivani M, Krishnan A, Aggarwal PK and Gupta SK: Prevalence of osteoarthritis of knee among elderly persons in urban slums using American college of rheumatology (ACR) criteria. J Clin Diagn Res 8: JC09-JC11, 2014.

12. Peterfy CG, Guermazi A, Zaim S, Tirman PF, Miaux Y, White D, Kothari M, Lu Y, Fye K, Zhao S and Genant HK: Whole-organ magnetic resonance imaging score (WORMS) of the knee in osteoarthritis. Osteoarthritis Cartilage 12: 177-190, 2004.

13. Hayashi D, Felson DT, Niu J, Hunter DJ, Roemer FW, Aliabadi P and Guermazi A: Pre-radiographic osteoarthritic changes are highly prevalent in the medial patella and medial posterior femur in older persons: Framingham OA study. Osteoarthritis Cartilage 22: 76-83, 2014.

14. Duncan RC, Hay EM, Saklatvala J and Croft PR: Prevalence of radiographic osteoarthritis-it all depends on your point of view. Rheumatology (Oxford) 45: 757-760, 2006.

15. Duncan R, Peat G, Thomas E, Wood L, Hay E and Croft P: How do pain and function vary with compartmental distribution and severity of radiographic knee osteoarthritis? Rheumatology (Oxford) 47: 1704-1707, 2008.

16. Amin S, Baker K, Niu J, Clancy M, Goggins J, Guermazi A, Grigoryan M, Hunter DJ and Felson DT: Quadriceps strength and the risk of cartilage loss and symptom progression in knee osteoarthritis. Arthritis Rheum 60: 189-198, 2009.

17. Rehan Youssef A, Longino D, Seerattan R, Leonard T and Herzog W: Muscle weakness causes joint degeneration in rabbits. Osteoarthritis Cartilage 17: 1228-1235, 2009.

18. Szebenyi B, Hollander AP, Dieppe P, Quilty B, Duddy J, Clarke S and Kirwan JR: Associations between pain, function, and radiographic features in osteoarthritis of the knee. Arthritis Rheum 54: 230-235, 2006.

19. Hunter DJ, Zhang Y, Niu J, Goggins J, Amin S, LaValley MP, Guermazi A, Genant H, Gale D and Felson DT: Increase in bone marrow lesions associated with cartilage loss: A longitudinal magnetic resonance imaging study of knee osteoarthritis. Arthritis Rheum 54: 1529-1535, 2006.
20. Peat G, Duncan RC, Wood LR, Thomas E and Muller S: Clinical features of symptomatic patellofemoral joint osteoarthritis. Arthritis Res Ther 14: R63,2012.

21. van Eijden TM, Kouwenhoven E, Verburg J and Weijs WA: A mathematical model of the patellofemoral joint. J Biomech 19: 219-229, 1986

22. Reilly DT and Martens M: Experimental analysis of the quadriceps muscle force and patello-femoral joint reaction force for various activities. Acta Orthop Scand 43: 126-137, 1972.

23. Froimson MI, Ratcliffe A, Gardner TR and Mow VC: Differences in patellofemoral joint cartilage material properties and their significance to the etiology of cartilage surface fibrillation. Osteoarthritis Cartilage 5: 377-386, 1997.

24. Mansuripur PK, Deren ME, Hayda R and Born CT: Initial management of ankle fractures in the overweight and obese: The providence pinch. J Emerg Med 47: 561-564, 2014

25. Gudbergsen H, Lohmander LS, Jones G, Christensen R, Bartels EM, Danneskiold-Samsøe B, Bliddal H and Boesen M: Correlations between radiographic assessments and MRI features of knee osteoarthritis-a cross-sectional study. Osteoarthritis Cartilage 21: 535-543, 2013.

26. Novais EN and Millis MB: Slipped capital femoral epiphysis: Prevalence, pathogenesis, and natural history. Clin Orthop Relat Res 470: 3432-3438, 2012.

27. Felson DT: Osteoarthritis as a disease of mechanics. Osteoarthritis Cartilage 21: 10-15, 2013.

28. Ulrich-Vinther M, Maloney MD, Schwarz EM, Rosier R and O'Keefe RJ: Articular cartilage biology. J Am Acad Orthop Surg 11: 421-430, 2003

29. Benichou OD, Hunter DJ, Nelson DR, Guermazi A, Eckstein F, Kwoh K, Myers SL, Wirth W and Duryea J; Osteoarthritis Initiative Investigators: One-year change in radiographic joint space width in patients with unilateral joint space narrowing: Data from the Osteoarthritis Initiative. Arthritis Care Res (Hoboken) 62: 924-931, 2010.

30. Spector TD, Hart DJ, Byrne J, Harris PA, Dacre JE and Doyle DV: Definition of osteoarthritis of the knee for epidemiological studies. Ann Rheum Dis 52: 790-794, 1993.

31. Link TM, Steinbach LS, Ghosh S, Ries M, Lu Y, Lane N and Majumdar S: Osteoarthritis: MR imaging findings in different stages of disease and correlation with clinical findings. Radiology 226: 373-381, 2003.

32. Baranyay FJ, Wang Y, Wluka AE, English DR, Giles GG, Sullivan RO and Cicuttini FM: Association of bone marrow lesions with knee structures and risk factors for bone marrow lesions in the knees of clinically healthy, community-based adults. Semin Arthritis Rheum 37: 112-118, 2007.

33. Felson DT, McLaughlin S, Goggins J, LaValley MP, Gale ME, Totterman S, Li W, Hill C and Gale D: Bone marrow edema and its relation to progression of knee osteoarthritis. Ann Intern Med 139: 330-336, 2003.

34. Roemer FW, Guermazi A, Javaid MK, Lynch JA, Niu J, Zhang Y, Felson DT, Lewis CE, Torner J and Nevitt MC; MOST Study investigators: Change in MRI-detected subchondral bone marrow lesions is associated with cartilage loss: The MOST Study. A longitudinal multicentre study of knee osteoarthritis. Ann Rheum Dis 68: 1461-1465, 2009.

35. Bellamy N: The WOMAC knee and hip osteoarthritis indices: Development, validation, globalization and influence on the development of the AUSCAN hand osteoarthritis indices. Clin Exp Rheumatol 23 (5 Suppl 39): S148-S153, 2005.

36. Hanna FS, Bell RJ, Davis SR, Wluka AE, Teichtahl AJ, O'Sullivan R and Cicuttini FM: Factors affecting patella cartilage and bone in middle-aged women. Arthritis Rheum 57: 272-278, 2007

37. Teichtahl AJ, Wluka AE, Proietto J and Cicuttini FM: Obesity and the female sex, risk factors for knee osteoarthritis that may be attributable to systemic or local leptin biosynthesis and its cellular effects. Med Hypotheses 65: 312-315, 2005. 